Provided for non-commercial research and education use. Not for reproduction, distribution or commercial use.

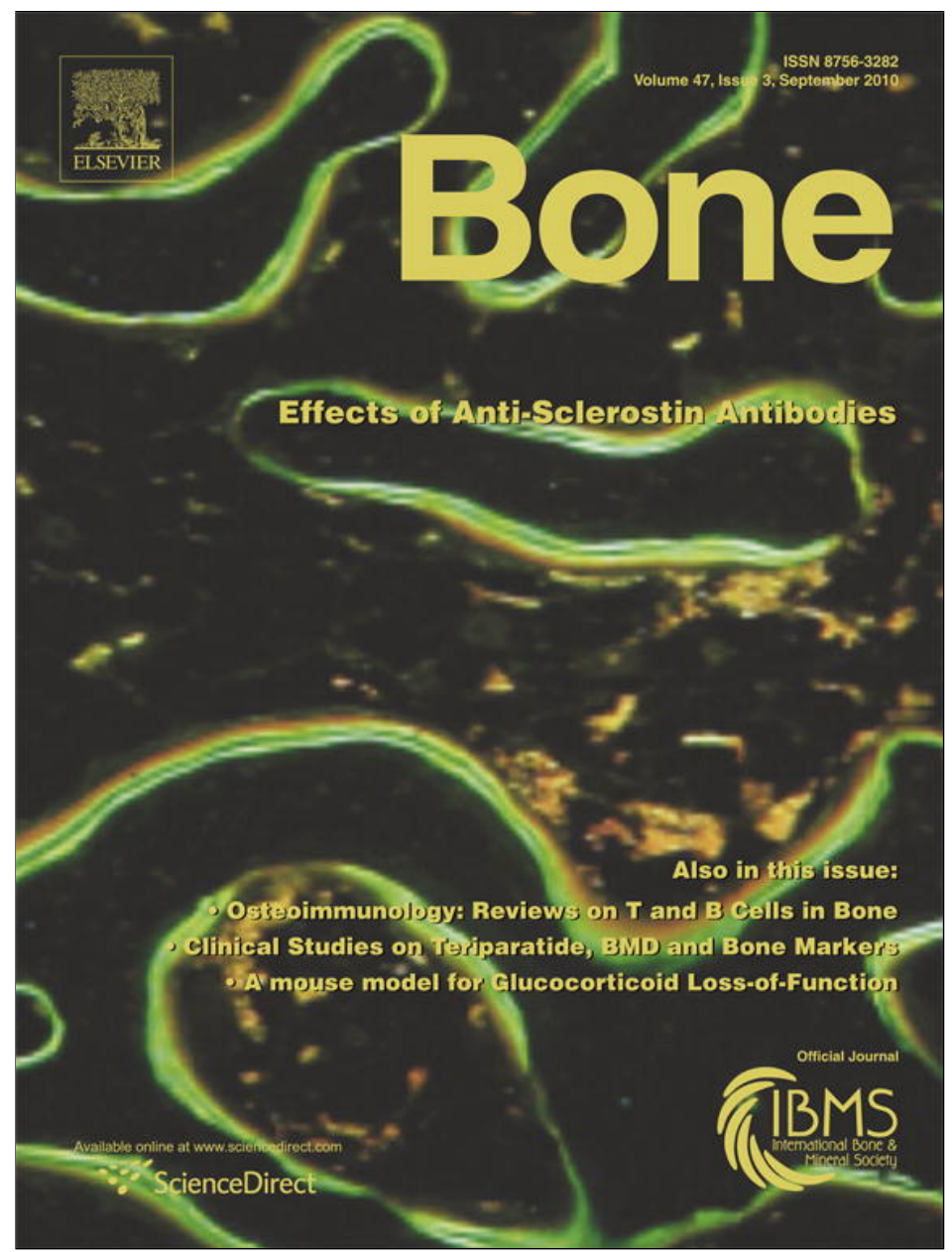

This article appeared in a journal published by Elsevier. The attached copy is furnished to the author for internal non-commercial research and education use, including for instruction at the authors institution and sharing with colleagues.

Other uses, including reproduction and distribution, or selling or licensing copies, or posting to personal, institutional or third party websites are prohibited.

In most cases authors are permitted to post their version of the article (e.g. in Word or Tex form) to their personal website or institutional repository. Authors requiring further information regarding Elsevier's archiving and manuscript policies are encouraged to visit:

http://www.elsevier.com/copyright 


\title{
Structural analysis of the human tibia in men with spinal cord injury by tomographic (pQCT) serial scans
}

\author{
Jörn Rittweger ${ }^{\mathrm{a}, \mathrm{b}, *}$, Vicky L. Goosey-Tolfrey ${ }^{\mathrm{a}, \mathrm{c}}$, Gustavo Cointry ${ }^{\mathrm{d}}$, José Luis Ferretti ${ }^{\mathrm{d}}$ \\ a Institute for Biomedical Research into Human Movement and Health, Manchester Metropolitan University, John Dalton Tower, Oxford Road, Manchester, M1 5GD, UK \\ ${ }^{\mathrm{b}}$ Institute of Aerospace Medicine, Department of Space Physiology, German Aerospace Center, Linder Höhe 1, D-51147 Cologne, Germany \\ c The Peter Harrison Centre for Disability Sport, School of Sport, Exercise and Health Sciences, Loughborough University, Epinal Way, Loughborough, UK \\ ${ }^{\mathrm{d}}$ Center of P-Ca Metabolism Studies (CEMFoC), National University of Rosario, Argentina
}

\section{A R T I C L E I N F O}

\section{Article history:}

Received 7 February 2010

Revised 30 April 2010

Accepted 18 May 2010

Available online 24 May 2010

Edited by: D. Burr

\section{Keywords:}

Immobilization

Bone biomechanics

Disuse-induced osteopenia

Bone density

Bone geometry

\begin{abstract}
A B S T R A C T
Spinal cord injury (SCI), as a primarily neurological disorder that causes muscular atrophy, is well known to be associated with sub-lesional bone losses. These losses are more pronounced from epiphyseal than from diaphyseal regions. We hypothesized that this discrepancy may be explained by anatomical variation in endocortical circumference.

Nine men who had attracted SCI 9 to 32 (mean 21.4) years prior to study inclusion were matched to able bodied control ( $\mathrm{Ctrl}$ ) people by age, height and weight. Serial scans by peripheral quantitative computed tomography were obtained from the tibia at steps corresponding to 5\%-steps of the tibias length (s05 to s95, from distal to the proximal end of the tibia).

As expected, SCI people had lower total bone mineral content (vBMC.tot) than able bodied control people $(P<0.001$ at all sites). This group difference $(\triangle \mathrm{vBMC}$.tot $)$ was more pronounced at the distal and proximal tibia than in the shaft $(P<0.001)$, and it amounted to $51 \%$ at s05, to $22 \%$ at $s 40$, and to $47 \%$ at s95. Both endocortical and periosteal circumference were better predictors of $\triangle$ vBMC.tot $\left(R^{2}=0.98\right.$ and $R^{2}=0.97$, respectively; $P<0.001$ in both cases) than vBMC.tot $\left(R^{2}=0.58, P<0.001\right)$, suggesting that anatomical variation in geometry, rather than in bone mass can explain differential rates of bone loss after SCI. Moreover, the s04:s38 ratio in vBMC.tot was found to be 1.00 (95\% confidence interval: $0.95-1.05)$ in the Ctrl group, and 0.63 in the $\mathrm{SCI}$ group $(P<0.001,95 \%$ confidence interval: $0.54-0.68$ ).

These findings offer a rationale to account for the discrepancy between epiphyseal and diaphyseal bone losses following SCI. The suggestion is that the bone adaptive responses involved are limited in time, and that the reduced surface:volume ratio constitutes a limit within the available time window, in particular in the diaphysis. Finally, the drastically reduced s04:s38 vBMC.tot ratio observed in the SCI group in this study provides a rationale to scrutinize this Capozza index also in other studies as a general indicator of immobilisation-induced bone loss. Crown Copyright @ 2010 Published by Elsevier Inc. All rights reserved.
\end{abstract}

\section{Introduction}

Spinal cord injury (SCI) leads to a profound muscular atrophy [1] and to a loss of bone tissue in the paralyzed limbs [2,3]. The consequence of this is reduced bone strength, and an increased risk of fractures in SCI patients [4-6], despite the fact that their risk of trauma is reduced. The rate of bone loss is initially rapid and slows down later on after $\mathrm{SCI}$, to reach a new steady state after 3-5 years [7,8]. The important question therefore arises, what mechanisms are involved in the cessation of bone loss at this time?

Bones respond to mechanical stimuli $[9,10]$. The mechanostat theory proposes peak strains as the central variable in a negative feed back control system [11]. Biomechanical analyses suggest that the largest

* Corresponding author. Institute of Aerospace Medicine, Linder Höhe 1, D51147 Cologne, Germany. Fax: +49220361159.

E-mail address: joern.rittweger@dlr.de (J. Rittweger). forces within our skeleton arise from muscle action [12,13], and it is therefore understandable that differences in musculature can account for inter-individual variation in bone strength [14-16]. It seems therefore reasonable to explain SCl-related bone losses by the absence of forceful muscle contractions, as a consequence of the neurological disorder. However, this explanation, at least on its own, cannot account for the observation that the tibia's epiphyses lose approximately $50 \%$ of the bone mineral content (BMC), whilst only $30 \%$ of the diaphyseal $\mathrm{BMC}$ is lost [7].

It has recently been suggested that bone losses from the human tibia are mainly caused by endocortical resorption in response to experimental bed rest [17]. In the latter study, the variation of bone losses within the human tibia could well be explained by anatomical variation in endocortical surface [17]. This is suggestive of the endocortical stratum, a zone that has particularly high bone turn-over $[18,19]$, to constitute an "active zone" for bone adaptive processes. One could therefore expect that the surface:volume ratio of the endocortical 
stratum will impose a limiting factor for bone adaptation. Accordingly bone adaptation would require longer time at sites with lower surface: volume ratio. Crucially, when time itself is limited, one should expect that sites with smaller endocortical surface should depict smaller bone losses than sites with larger endocortical surface.

Based on this theoretical framework, we hypothesized that the reduction in diaphyseal bone mass, as observed in people after spinal cord injury, is in a direct relationship to endocortical circumference. We have therefore studied the human tibia in $\mathrm{SC}$ participants and able bodied control people by serial tomographic scans, a technique that has recently been described [20]. The idea was to test the aforementioned hypothesis by comparing endocortical circumference and the SCI-related bone deficit in the various sections obtained.

\section{Materials and methods}

\section{Study participants}

Ten male participants with paraplegia due to spinal cord injury were recruited through the wheelchair basketball, tennis and hand cycling National Governing Sporting Bodies for paraplegic people. Data from one participant had to be excluded because of a leg positioning error, leaving nine SCI participants, the data of which are presented here. All participants undertook daily living tasks independently, and were using a hand-rim wheelchair for all their daily movement requirements. This was determined from a self-recorded weekly activity log. All participants were considered to be trained, having competed regularly in wheelchair sporting activities at a National level. The participant disability descriptive characteristics are shown in Table 2. Eight participants reported permanent loss of both sensory and motor function which was considered as a complete lesion. One participant had an incomplete lesion, which implied preservation of sensory or motor function below the level of injury, including the lowest sacral segments. Able bodied control participants (Ctrl) were matched by body height, weight and age to the SCI people. Ctrl participants were recruited among members of staff of the Alsager campus of the Manchester Metropolitan University. They were devoid of any known musculoskeletal disorder or major disease, and all of them participated regularly in recreational physical activity, such as running, cycling, swimming football, basketball and volley ball.

\section{Scanning procedures}

Bone scans were taken with a XCT 2000 (Stratec, Pforzheim, Germany). The image resolution was set to $0.5 \mathrm{~mm}$ edge length (software option "Voxel Size"). The tibia length (L.Tib) was identified as the distance between the medial malleolus and the medial knee

Table 1

List of pQCT variables and their acronyms.

\begin{tabular}{ll}
\hline Ar.Bo & Bone cross sectional area \\
\hline Ar.Ct & Cortical cross sectional area \\
Ar.Epi & Epiphyseal cross sectional area \\
Ar.MB & Combined muscle and bone cross sectional area \\
Ar.tot & Limb's total cross sectional area \\
cBMC & Cumulative BMC for entire tibia (in grams) \\
EcC & Endocortical circumference \\
L.Tib & Tibia length \\
MI.p & Polar moment of inertia \\
PsC & Periosteal circumference \\
vBMC & Volumetric bone mineral content \\
vBMC.tot & Total vBMC \\
vBMD & Volumetric bone mineral density \\
vBMD.Ct & Cortical vBMD \\
\hline
\end{tabular}

Acronyms are in accordance with the recommendations for high-resolution pQCT by ASBMR (http://nomenclature.bb.asbmr.org).
Table 2

Survey of the SCI participants.

\begin{tabular}{llllll}
\hline Participant & $\begin{array}{l}\text { Current } \\
\text { age }\end{array}$ & $\begin{array}{l}\text { Age at } \\
\text { SCI }\end{array}$ & $\begin{array}{l}\text { Duration of SCI } \\
\text { (years) }\end{array}$ & $\begin{array}{l}\text { Lesion } \\
\text { level }\end{array}$ & $\begin{array}{l}\text { Complete/ } \\
\text { incomplete }\end{array}$ \\
\hline 1 & 39 & 19 & 20 & T7-T8 & Complete \\
2 & 45 & 35 & 10 & T6 & Complete \\
3 & 33 & 20 & 13 & T8-T10 & Complete \\
4 & 40 & 18 & 22 & T7 & Complete \\
5 & 41 & 25 & 16 & L1 & Incomplete \\
6 & 32 & 23 & 9 & T5 & Complete \\
7 & 49 & 17 & 32 & T5 & Complete \\
8 & 43 & 19 & 24 & T5/6 & Complete \\
9 & 31 & 17 & 14 & T12 & Complete \\
\hline
\end{tabular}

All SCI participants were motor complete, but SCI participant 5 had some sensory function left. All SCI participants were wheel chair bound.

a Indicates flaccid paralysis.

joint cleft (Table 1). Sequential scans were then taken in steps of $5 \%$ of the tibia length, starting from distal (s05) to the proximal (s95) part of the tibia. Distal and proximal tibia joint surfaces were identified by appropriate scout viewing procedures as described before $[15,16]$. Because our XCT model did not allow for measurements of the entire lower leg, ten sequential scans were taken starting from the distal tibia joint surface, and ten sequential scans were taken from the proximal tibia plateau. Hence, the 10th scan from the proximal end and the 10th scan from the distal end of the tibia ought to be in the same place, namely at $50 \%$ of the tibia's length (s50). Identity of both s50 scans was ascertained by applying a mark on the skin at the actual measurement site (using the laser beam of the XCT) after the 10th scan of the first set of images, and by verifying its position during the second set of images. Moreover, analysis of the duplicate s50 scans from both sets of images yielded no difference between values ( $0.41>$ $P>0.92$ ) for total bone area (Ar.Bo), total BMC (vBMC.tot), cortical BMC (vBMC.Ct), and periosteal and endocortical circumferences (PsC and $\mathrm{EcC}$, respectively). Furthermore, variation between s50 values from the proximal and distal sets of images was rather small, with variation coefficients (CV) ranging between $1.9 \%$ and $5.5 \%$, altogether suggesting good positioning of the two s50 scans. Accordingly, we have used the average of the two s50 measurements for further analysis.

\section{Image analysis}

pQCT images were analysed with the integrated XCT software in its version 6.00 , using the "automated analysis" tool. Given the very thin cortices in the epiphyses of $\mathrm{SCl}$ people, a comparatively low detection threshold had to be applied. Therefore, regions of interest (ROIs) were placed tightly around each bone. ROIs were then minimized using the " $m$ " option, and then slightly enlarged using the "+" option within the integrated XCT software. This resulted in a very tight fitting of the ROIs around the bone, and thus minimized contamination of the results by soft tissue contributions. Different peeling thresholds were tested, and it was found that a threshold of $120 \mathrm{mg} / \mathrm{cm}^{3}$ could adequately separate bone and soft tissue in all scans used for analysis. Accordingly, this threshold was then used to assess total bone area Ar.Bo and vBMC.tot. It should be understood that a threshold as low as $120 \mathrm{mg} / \mathrm{cm}^{3}$ will lead to a small overestimation of Ar.Bo (about 5\% in this case [21]), which depends on the periosteal circumference and pixel edge length. On the other hand, it was essential in this study to analyse all sectional images with one same threshold, and, more importantly, a low threshold will lead to an accurate assessment of vBMC. Accordingly, the main focus of this analysis is upon vBMC values.

Cortical vBMC (vBMC.Ct) was computed with a threshold of $650 \mathrm{mg} / \mathrm{cm}^{3}$, using the "peel mode 1" option in the XCT software. The 
threshold of $650 \mathrm{mg} / \mathrm{cm}^{3}$ was chosen for this computational step because it has been demonstrated to be accurate for the detection of cortical bone [22], and because we have already applied it successfully in the past [23]. Trabecular vBMC (vBMC.tb) was subsequently computed as vBMC.tot-vBMC.Ct. The polar moment of inertia (MI.p) was identified as the variable IP_CRT_A from the resulting XCT loopdatabase. The axial moments of inertia yielded results that were very similar to MI.p and are therefore not reported here. PsC and EcC were identified as PERI_C and ENDO_C, respectively, from the loop-database. It should be noted that these variables arise from a so-called ring-model, i.e. it is assumed that the bone tissue is cylindrically distributed around a central axis. These variables therefore are more reflective of the average distance of the bone envelopes from the central axis, rather than an accurate measurement of the anatomical surface size of the tibia. Cortical vBMD (vBMD.Ct) was adjusted for the partial volume effect as previously described [21]. This procedure fails when cortical thickness is less than twice the image resolution, and cortical bone data (EcC, vBMD.Crt, MI.p) were analyzed for sites between s15 and s85 only to prevent such failure.

\section{Statistical analyses and further computations}

Anthropometric data were compared between groups with Student's $t$-test. For statistical analysis of pQCT data, linear mixed effect (LME) models were generated for each variable with site and group as fixed effects and participant ID as random effect. Variances were allowed to differ between participants and site, and LME models were optimized according to Akaike's information criterion (see p.353 and p.652 in [24]). Significant effects were followed up by a-priori contrasts, comparing the group difference for each site to the s05 site, and to the $\mathrm{s} 15$ site for cortical bone variables. The s05 site was chosen to represent the epiphysis so that effects by SCI that would affect the diaphysis differentially can be picked up by site $\times$ group interaction effects in the LME models. The s15 site was chosen as the cortical site where vBMC.tot is smallest, in line with the view that it is strained almost purely in compression [20]. Therefore, s15 is an ideal site of reference to monitor differential effects of SCI upon bending stiffness and compressive strength.

In order to test our primary hypothesis, namely that anatomical variation in circumference can account for the magnitude of SCIrelated bone deficits, we generated a new variable, $\triangle \mathrm{vBMC}$.tot, for each site as the difference between the mean values. We then performed correlation analysis between $\triangle \mathrm{vBMC}$.tot and the EcC. We decided to use EcC values from the Ctrl group for this (rather than EcC values from the SCI group or the group difference) because our hypothesis relates circumference at the onset of SCI. The expectation was that sites with greater circumference, and thus with larger endocortical transition zone, would also depict a greater deficit. Moreover, and since results obtained in this study are compatible with the view that there may also be periosteal bone losses, the same analysis has also been performed for PsC.

Statistical analyses were carried out in the $R$ statistical environment (version 2.9.2, www.r-project.org). Data are presented as means and their standard errors, if not indicated otherwise. A level of 0.05 for $\alpha$ was chosen for statistical significance.

Finally, BMC (in $\mathrm{mg} / \mathrm{mm}$ ) was integrated over the 19 different slices, whilst accounting for the inter-slice distance, to yield the tibia's cumulative BMC (cBMC, in grams). This was done for total BMC, as well as for the cortical and the trabecular portion. Moreover, in order to validate the suggestion that the vBMC.tot ratio between s38 and s04 can be used as a clinical indicator of tibial bone losses [20], s38values have been computed by linear interpolation between s35 and $\mathrm{s} 40$, and by linear extrapolation from s10 and s05 values. This was done for each individual, and $95 \%$-confidence intervals (95\%-CI) have been computed per group for the s04:s38 ratio as well as for the s05: s40 ratio, with the latter being obtained for each single individual.

\section{Results}

Study cohort

SCI participants had their lesions between the levels of L1 and T5 (Table 2). The SCI had occurred at an average age of 21.4 years (range $17-35$ years). This was on average 17.8 years prior to study inclusion (range 9-32 years). The anthropometric data of both groups at the time of study are given in Table 3. No group difference was found in these data $(P>0.26)$.

\section{Qualitative bone findings}

Typical examples of pQCT images are displayed in Fig. 1. It was quite obvious when viewing those images that trabecular bone losses had occurred at epiphyseal sites, and that the cortical shell was generally thinner in SCI people than in Ctrl (Fig. 1A). However, it seemed that the bone mineral density of compact bone was not largely reduced in $\mathrm{SCI}$ people. Rather, three out of the nine SCI people depicted intra-cortical portions of trabecular bone, with a layer of compact bone that could well correspond to the endocortical layer at the time of the SCI (Fig 1B). Where such intra-cortical trabecular portions appeared, there they were observed over several sequential tibia levels, namely from s50 to s65 in SCI participant 2, from s35 to 860 in SCI participant 6, and from s20 to s75 in SCI participant 7. Moreover, it was apparent that diaphyseal sites depicted greater portions of trabecular bone in SCI participants as compared to their able bodied control persons.

\section{pQCT results}

A complete set of pQCT data was obtained from each participant included in this analysis. Significant group effects, site effects, and group $\times$ site interaction were found for vBMC .tot $(P<0.001$ in all instances). In keeping with previous research [20], vBMC.tot was comparable between s05, s30 and s35 (no main effect for site), but different between $\mathrm{s} 05$ and all other sites ( $P$-values between $<0.001$ and 0.03 , see Fig. 2). Significant interaction effects between group and site revealed a virtually constant difference for all sites between s10 and s75 $(P<0.001$ for all sites), and increasingly larger differences between s75 and s95 $(P<0.001)$. The s05:s40 ratio in vBMC.tot was 0.94 (95\%-CI: 0.89-0.99) in Ctrl and $0.61(P<0.001,95 \%-C I: 0.54-0.68)$ in SCI, and the s04:s38 ratio was 1.00 (95\%-CI: $0.95-1.05)$ in $\mathrm{Ctrl}$ and $0.63(P<0.001,95 \%-\mathrm{CI}$ : $0.55-0.71)$ in SCI. Importantly, there was not any overlap of values between groups for these ratios.

Both vBMC.Ct and vBMC.tb yielded main effects for site and group $(P<0.001$ in all instances) and also significant interaction effects $(P<0.001)$. Interaction effects were significant between all sites from s10 to $s 90$ for vBMC.Ct $(0.0001<P<0.039)$ and between all sites from s15 to s90 for vBMC.tb $(0.0001<P<0.002$, see Fig. 2$)$. Moreover, vBMC.tb was greater in SCI than in Ctrl at sites s30, s35 and s $40(0.018<P<0.041)$, and non-significantly elevated at sites s45 and s50 $(P=0.065$ in both cases).

There were also significant main and interaction effects for PsC $(P<0.001$ in both instances, see Fig. 3$)$. Post hoc analysis yielded that group $\times$ site interaction was significant for all sites between $s 20$ and s75 $(0.0013<P<0.0127)$.

\section{Table 3}

Anthropometric variables.

\begin{tabular}{llllll}
\hline & $N$ & Age [years] & Height $[\mathrm{cm}]$ & Mass $[\mathrm{kg}]$ & L.Tib $[\mathrm{cm}]$ \\
\hline $\mathrm{SCl}$ & 9 & $39.2(6.2)$ & $178.8(4.3)$ & $76.9(9.0)$ & $38.5(1.9)$ \\
Ctrl & 9 & $39.6(7.8)$ & $177.2(2.6)$ & $77.9(9.0)$ & $37.6(1.2)$ \\
$P$-value & & 0.92 & 0.36 & 0.82 & 0.26 \\
\hline
\end{tabular}

No group difference was found in age, height, body mass or tibia length (L.Tib). 


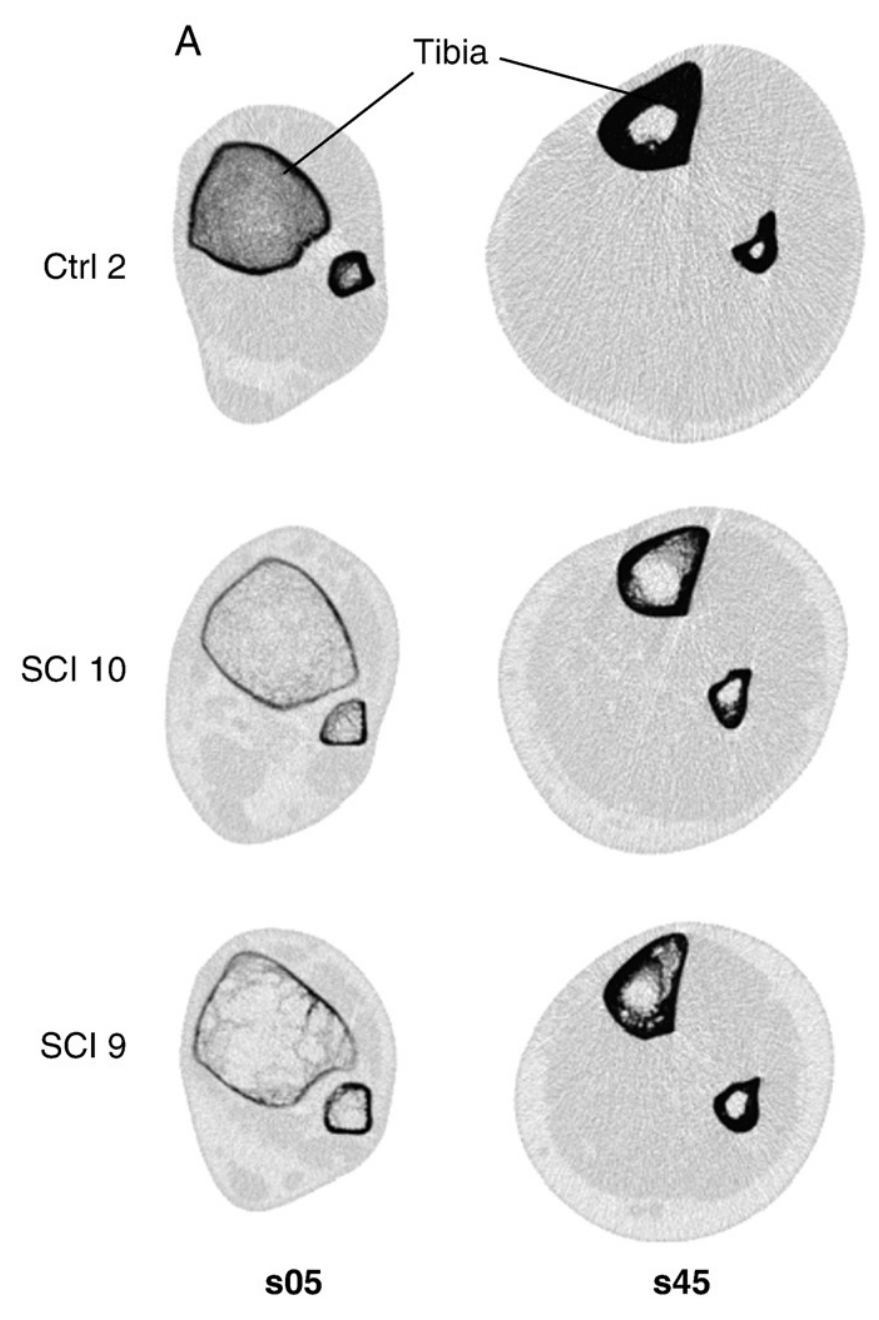

$B$
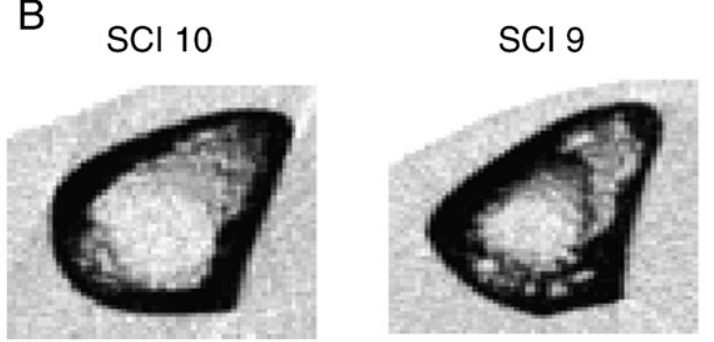

Fig. 1. (A) Survey of typical pQCT images in an able bodied control person (Participant Ctrl 04, upper row) and two participants with spinal cord injury (SCI 10 and SCI 9 in the middle and lower row, respectively). For the epiphyseal scan at site s05 (meaning at 5\% of the tibia's length from its distal end) it is apparent that SCI participants have thinner cortices and also reduced trabecular bone mineral density. Cortical thinning can likewise be observed at diaphyseal sites in the SCI participants, as displayed here for 545 (i.e. at $45 \%$ of the tibia's length). In addition, the images demonstrate the presence of trabecular bone where would normally expect compact bone. This was consistently observed in the frontal aspect of the tibia, and occasionally also in the dorsal aspect of the tibial cortex (e.g. here in SCI 7). (B) Greater magnification of the tibias from SCI participants at s45 in Fig. 1.A: The endocortical surface seems to be consistently eroded in SCI 10. By contrast, the image of SCI 9 suggests that the endocortical surface has prevailed, and that intra-cortical bone resorption may have predominated.

Main effects by site and group were discovered for EcC and vBMD. $\mathrm{Ct}(P<0.001$ in all instances $)$, but no significant interaction effect was found $(P=0.23$ and $P=0.61$, respectively). Likewise, there were significant main effects by site and group upon MI.p $(P<0.001)$, but no site $\times$ group interaction $(P=0.054)$.

The relationships between group difference in vBMC.tot $(\triangle \mathrm{vBMC}$.tot, dependant variable) and PsC and EcC (independent variable) have been analysed in Fig. 4. Correlation analysis yielded excellent fit of the linear regression models, with $R^{2}$ values for EcC and PsC of 0.98 and 0.97 , respectively $(P<0.001)$. Including both $\mathrm{PsC}$ and $\mathrm{EcC}$ in the model led to a fit that was marginally better than for PsC alone $(P=0.03)$, but no improvement was achieved over EcC alone $(P=0.1)$. Correlation analysis between EcC and PsC yielded an $R^{2}$ value of 0.97 , indicating statistical colinearity. When plotting $\triangle \mathrm{vBMC}$.tot versus the vBMC.tot values from the Ctrl group, a significantly inferior fit was obtained $\left(R^{2}=0.58, P<0.001\right)$, suggesting that anatomical variation in EcC and $\mathrm{PsC}$ is a better predictor of SCI-related bone deficit than anatomical variation in bone mass.

\section{Discussion}

Past studies had suggested that reduction in tibial bone mineral content after spinal cord injury is more pronounced in the epiphyses than in the diaphyses. This finding has been corroborated here, and more pronounced epiphyseal vs. diaphyseal deficits have been demonstrated as a general feature (Fig. 2). The s04:s38 ratio, which was 1.00 in the Ctrl group and 0.63 in the SCI group, highlights the general finding to propose this Capozza-index as an indicator of immobilization-related bone loss in the tibia. In addition, the present study also demonstrates that $\mathrm{vBMC}$ reduction, at least in absolute terms, is more pronounced in the proximal tibia than in the distal tibia, that periosteal circumference is clearly reduced in SCI people, whilst reductions in cortical vBMD are very moderate. Most importantly, SCI-related bone deficits seem to be well explicable by anatomical variation in periosteal and endocortical circumference. The latter finding has a couple of important implications.

Firstly, fractures are occurring quite commonly in the distal and proximal tibia, but are less frequent in the shaft [5]. It is obvious that this pattern can well be explained on the basis of our findings, namely predominant bone deficits at either end of the tibia. It had previously been thought that SCI-related bone losses would be largest in the most distal parts of the leg [25]. This is clearly not the case within the tibia, where deficits from the proximal epiphysis seem to be larger from its proximal than from its distal part (Fig. 3 and Table 4).

It is understood that the endocortical layer constitutes an active transition zone for bone adaptive processes [17,19,23]. One could speculate, based upon the group difference in PsC in this study, that there is also immobilization-related resorption on the periosteal envelope. As an alternative explanation for the smaller periosteal circumference in SCI we need to consider age-related periosteal apposition, known to occur in the femoral neck [26] and the tibia [27]. The latter study reports an increase by $0.6 \%$ per decade. This figure appears to be small in relation to the $5.8 \%$ group difference at $s 40$ in this study, which for the 21.4 years of time since SCI would equate in to a group difference of $2.7 \%$ per decade. Accordingly, the smaller periosteal circumference in the SCI participants' tibia may not entirely be caused by blunted periosteal expansion alone, but periosteal resorption could potentially also occur following SCI. If such periosteal resorption does occur, then our data suggest that it would be different from endocortical bone resorption in that periosteal resorption maintains a distinguished envelope. The suspected endocortical resorption, by contrast, followed an entirely different pattern, whereby some of the bone may have been left behind (transformation of formerly compact into trabecular bone tissue), or where trabecular bone was generated similar to ectopic bone formation (see Fig. 1B). At least this explanation could account for the increases in vBMC.tb in the SCI group (Fig. 3) in the presence of an overall deficit in vBMC.tot in that group.

It has been argued that the long bones' ends are mainly loaded in compression, whereas the shaft is experiencing considerable bending and torsional strains [20]. Quite possibly, SCI imposes differential reductions upon compressive versus torsional and bending loads. This could potentially explain the relative sparing of diaphyseal BMC [16] 

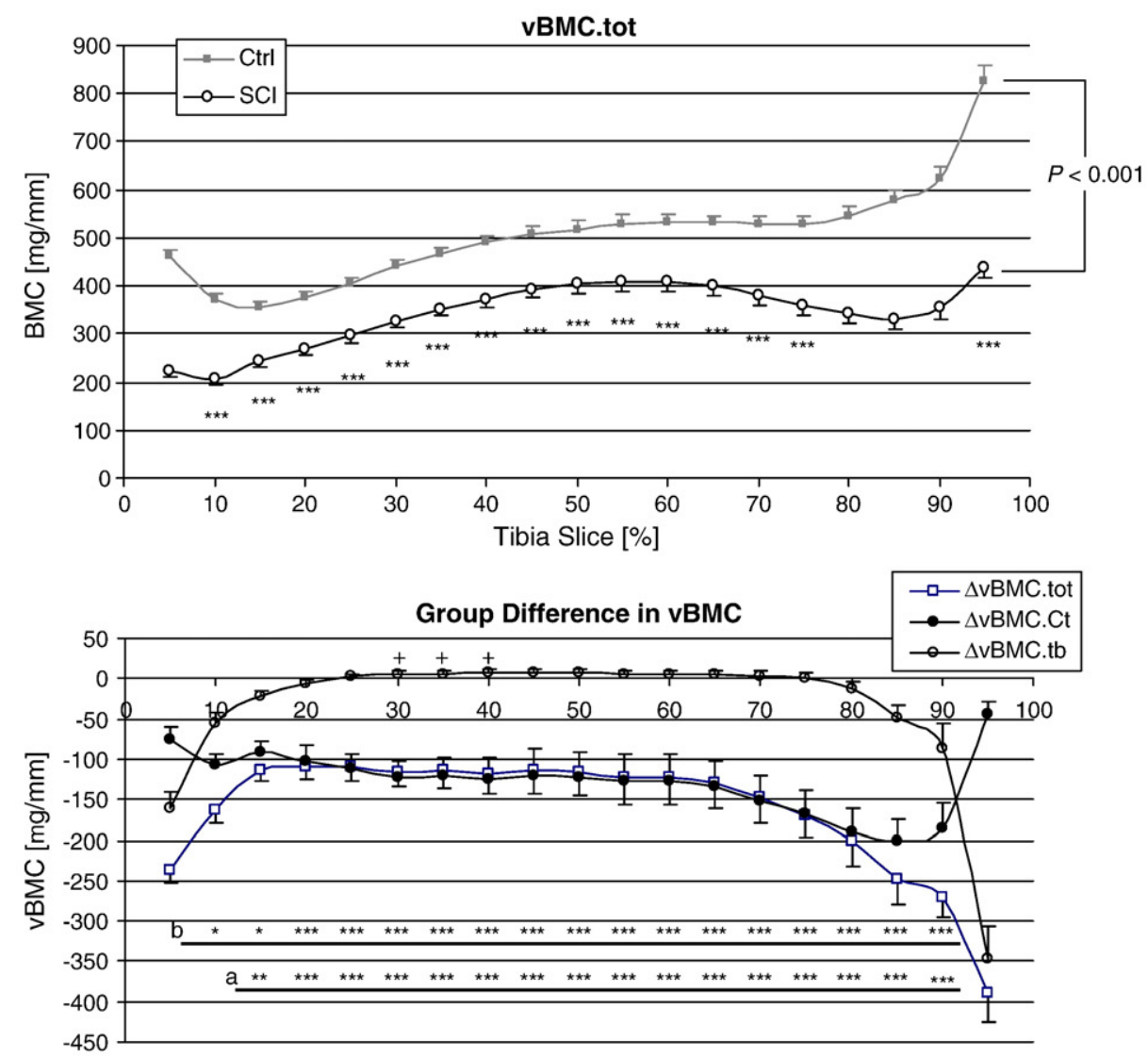

Tibia Slice [\%]

Cortical vBMD

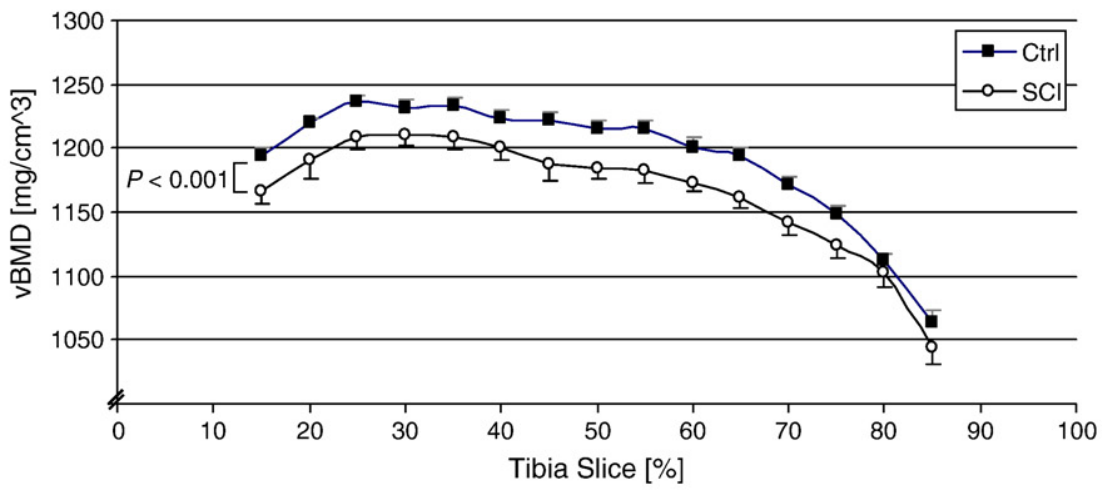

Fig. 2. Differences in bone mineral content (vBMC) and in cortical BMD between SCI participants and able-bodied control participants (means and SEM). Upper diagram: total vBMC (vBMC.tot) yielded a significant main effect for group $(P<0.001)$ and for site $(P<0.001)$, as well as group $\times$ site interactions $(P<0.001)$. Asterisks denote a-priori contrasts for a site $\times$ group interaction with ${ }^{* * *} P<0.001$. In the middle diagram, the group differences in total BMC $(\triangle \mathrm{vBMC}$.tot), cortical BMC $(\triangle \mathrm{vBMC} . \mathrm{Ct})$ and trabecular BMC $(\triangle \mathrm{BMC}$.tb) have been computed from the matched pairs, after significant group effects and group $\times$ site interactions had been found for these variables $(P<0.001$ in all instances). Interaction effects for (a) vBMC.tb and (b) vBMC.Ct have been displayed with ${ }^{*} P<0.05,{ }^{* *} P<0.01{ }^{* * *} P<0.001$. SCI participants had greater vBMC.tb at three sites with ${ }^{+} P<0.05$. The lower diagram shows cortical bone mineral density, for which significant group and site differences were found $(P<0.001$ in both instances), but no group $\times$ site interaction. Despite the significant group difference, it is evident from this Figure that reductions in cortical vBMD have only a minor contribution to the overall bone deficit in SCI people (please note broken y-axis in the lower panel). Error bars denote the standard error of the mean (SEM) for each group in the upper and lower diagram, and the SEM of the difference between matched pairs in the middle diagram.

(explanation A) [16]. However, the mottled cortical bone pattern (Fig. 2B), which was found in 3 out of $9 \mathrm{SCl}$ participants, and which is not observed in able bodied people (personal experience by the authors in more than 1000 people tested) constitutes strong evidence against explanation $\mathrm{A}$. This is because the endocortical remnant is comparatively inefficient to provide bending and torsional strength, implying impaired adaptation to bending and torsion. However, bending and torsion were held responsible by explanation A for diaphyseal sparing of bone, leading to a contradictio in re, which disproves model $\mathrm{A}$ as an explanation. Rather, endocortical bone resorption appears to be hampered by some unknown mechanisms in these SCI participants, and this seems to be the reason for the remnant layer.

It is important to consider the effect of time at this point. Of note, epiphyseal bone losses tend to level off more than twice as fast than diaphyseal losses [7]. Evidence suggests that bone adaptive processes cease after a certain period of time, e.g. by the phenomenon of cellular accommodation [28]. Moreover, osteocytic cell death, which is likely to occur in an environment with little or no mechanical strain at all [29], could lead to the loss of adaptive capability altogether in 


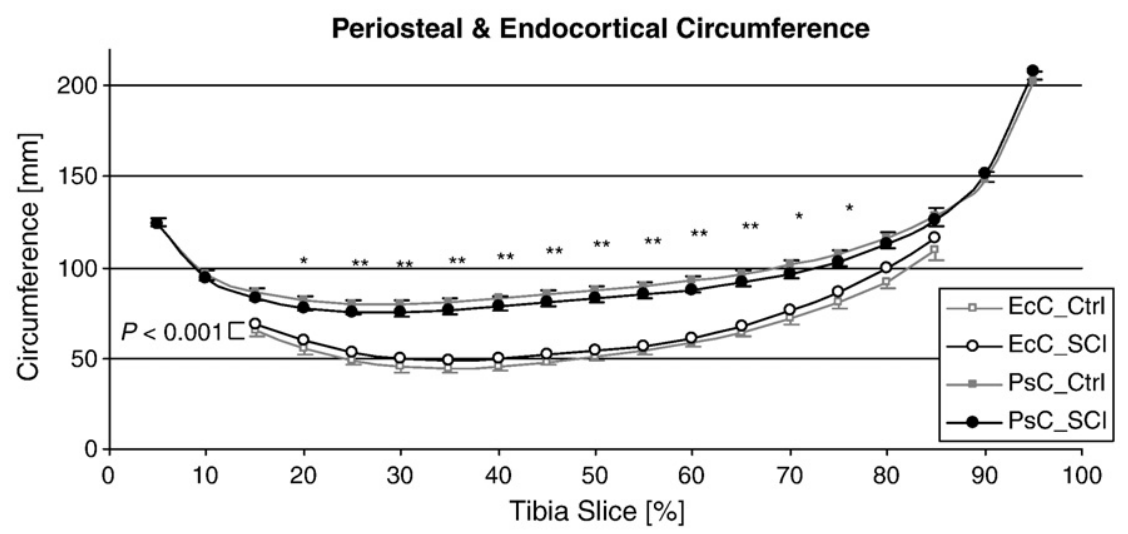

Group Differences

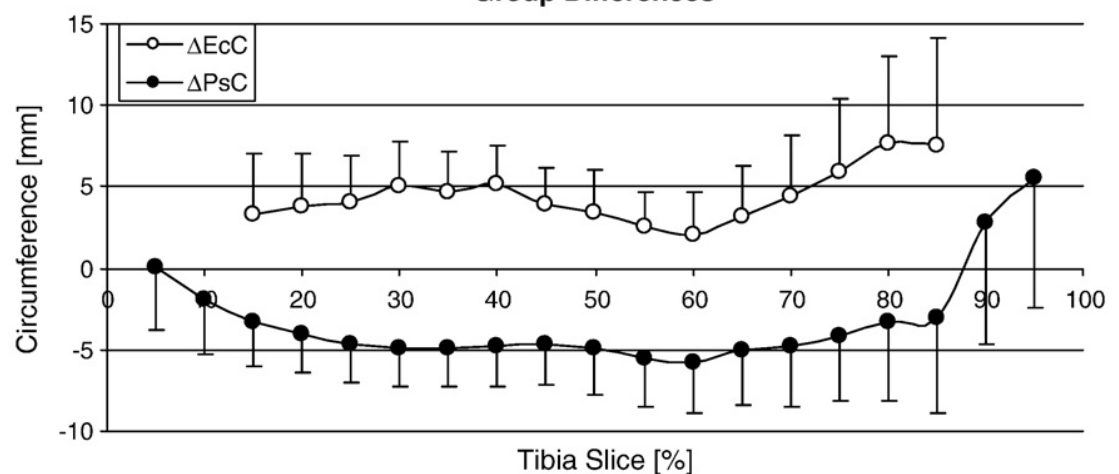

Fig. 3. Differences in diaphyseal design. Upper diagram: periosteal (PsC) and endocortical $(\mathrm{EcC})$ circumferences yielded a significant main effect for group $(P<0.001)$ and for site $(P<0.001)$, as well as group $\times$ site interactions $(P<0.001)$. Asterisks denote a-priori contrasts, for site $\times$ group interaction for $P s C$ with ${ }^{*} P<0.05$, ${ }^{* *} P<0.01$. The group differences in PsC and $\mathrm{EcC}(\triangle \mathrm{PsC}$ and $\triangle \mathrm{EcC}$, respectively) have been displayed in the lower diagram to allow an assessment of their magnitude. These variables have been computed as the difference between the matched pairs from the Ctrl group and from the SCI group. The distance between both curves illustrates the group difference in cortical thickness. Error bars denote the SEM for each group in the upper diagram, and the SEM of the difference between matched pairs in the lower diagram.

the bones of SCI patients. Indeed, muscle contractions induced by functional electrical stimulation in patients more than 10 years post $\mathrm{SCl}$ seems to elicit no [30] or only very small benefits in bone mass [31].

\section{BMC Difference vs. Circumference}

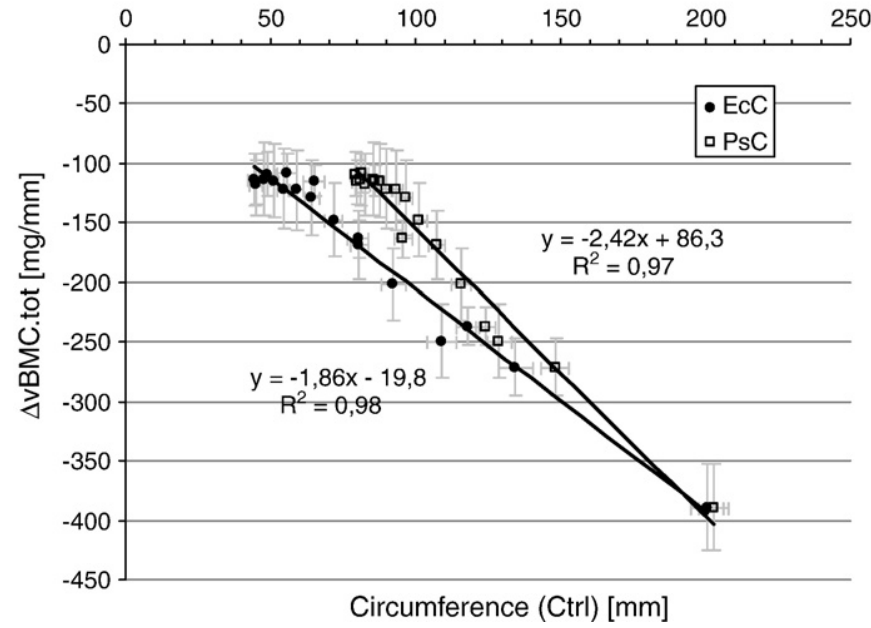

Fig. 4. Comparing group differences in vBMC.tot and periosteal and endocortical circumferences (PsC and EcC, respectively). Group differences in vBMC.tot were computed for each site ( $\mathrm{s} 05$ to s95) as the difference between the matched pairs from the $\mathrm{SCI}$ group and from the Ctrl group. As independent variables, PsC and EcC values from the Ctrl group were used. Results suggest that overall bone deficits in SCI participants can well be explained by anatomical variation in periosteal and endocortical envelope size.
Combining the above observations and ideas allows the following explanation to account for the striking dissociation between epiphyseal and diaphyseal bone losses in SCI patients: We suppose that (i) the signals for bone resorption become less effective with time, that (ii) the time permitted for adaptation is insufficient, in particular in the diaphyses, where (iii) the reduced surface:volume ratio restricts bone losses.

On a more practical level, we have utilized our data in order to give a survey of the amount and origin of SCI-related reductions in bone

Table 4

Quantification of SCI-related deficit in vBMC.

\begin{tabular}{clccc}
\hline & & \multicolumn{1}{l}{ Ctrl } & SCI & Difference \\
\hline Entire tibia & cBMC.tot $[\mathrm{g}]$ & $190.4(16.9)$ & $132.2(21.6)$ & $-58.2(30.8)$ \\
s5-s95 & cBMC.Ct [g] & $139.6(14.6)$ & $94.0(19.1)$ & $-45.6(26.8)$ \\
& cBMC.tb [g] & $50.8(6.7)$ & $38.1(4.8)$ & $-12.7(10.6)$ \\
& Volume [cm $\left.{ }^{3}\right]$ & $349.3(51.3)$ & $342.5(45.5)$ & $-6.8(81.9)$ \\
& & & & \\
Proximal and distal & cBMC.tot [g] & $63.7(6.6)$ & $36.4(6)$ & $-27.4(8.5)$ \\
s5-s15 and s85-s95 & cBMC.Ct [g] & $26.5(5.0)$ & $12.8(4.0)$ & $-13.6(6.2)$ \\
& cBMC.tb [g] & $37.7(5.0)$ & $23.5(3.8)$ & $-13.7(7.5)$ \\
& Volume [cm $\left.{ }^{3}\right]$ & $177.0(30.1)$ & $183.3(25.8)$ & $6.3(47.8)$ \\
Shaft only & & & & \\
s20-s80 & cBMC.tot [g] & $126.7(11.4)$ & $95.8(16.2)$ & $-30.9(23.0)$ \\
& cBMC.Ct [g] & $113.1(11.2)$ & $81.2(15.8)$ & $-31.9(22.4)$ \\
& cBMC.tb [g] & $13.6(2.4)$ & $14.6(1.9)$ & $1.0(3.9)$ \\
& Volume [cm $\left.{ }^{3}\right]$ & $172.3(23.0)$ & $159.2(22.6)$ & $-13.1(38.5)$ \\
\hline
\end{tabular}

Survey of the bone mass and volume accumulated over the entire tibia, over the shaft and over the tibia's endings. Computation of these values incorporated the inter-slice distance and thus gives an estimate of the SCI-related deficits over the entire tibia. Furthermore, total bone mass (vBMC.tot) has been separated into cortical (vBMC.Ct) and trabecular (vBMC.tb) portions. 
mass. As can be seen from the lower panel of Fig. 2, the group difference in cortical vBMD was very small, amounting to less than $3 \%$. This implies that by far the greatest part of the SCI-related bone deficit in the shaft is due to a deficit in tissue volume rather than to a reduction in tissue vBMD. As demonstrated in Table 4, approximately half of the SCI-related bone deficit predominates from the shaft, and the other half originates from the endings. Within the shaft, the average cortical bone mineral density in the SCI group was $1.17 \mathrm{~g} /$ $\mathrm{cm}^{3}$, and the group deficit in total volume amounted to $13.1 \mathrm{~cm}^{3}$ (Table 4). This deficit in volume arises from a "missing" space on the outside of the bone, and multiplication of the latter two values yields an estimated deficit of approximately $15.3 \mathrm{~g}$ that is "missing" on the periosteal envelope in the SCI group-which accounts for about half of the BMC deficit within the shaft (30.9 g, Table 4).

Within the tibia endings, approximately half of the bone deficit originates from the trabecular, and half from the cortical portion. It is probably prudent to assume that, as observed in the compact bone compartment of the shaft, there will be no large group differences in vBMD at the material level. If so, then this would imply that most of the bone deficit in the tibia's endings will be due to a reduction in the amount of bone tissue. A recent DXA-based study of former adolescent athletes suggests that trabecular bone sites tend to be more prone to bone losses than sites that are mainly composed of compact bone [32]. However, this study and several other CT-based studies clearly demonstrate that any losses from trabecular site predominate from the cortical portion [33,34], most likely from its endocortical aspect $[17,23]$. Future studies will have to demonstrate whether the s04:s38 BMC ratio (Capozza index) can be used as a diagnostic tool to monitor transients of bone loss.

The main limitation of this study is its cross-sectional design. This would be particularly problematic if SCI people were still in the process of losing bone. However, SCI had occurred 9 years or even more prior to study inclusion, and it is therefore to be expected that a new steady state had been reached. Eser et al. [7] have reported steady state values for SCI-related BMC-reductions to be $-58 \%$ and by $-25 \%$ at the tibial s04 and the s38 sites, respectively. Results of this study indicate group differences by $51 \%$, by $22 \%$ and by $47 \%$ for the s 05 , the s40 and the s95 sites, respectively. Whilst our figures are somewhat smaller than the aforementioned, we feel that they still suggest steady state conditions in our cohort, and also generalizability of our results. As a second concern that is generally associated with cross-sectional studies we have to consider selection bias. Whilst it would be cynical to discuss such effects in relation to SCI people, we would argue that any selection bias was effectively reduced by careful selection of control participants. Finally, this study focuses on the tibia and neglects the fibula. However, there are currently no good models available for the force transmission between tibia and fibula. Moreover, it is hard to see how the fibula could have affected the most important finding of this, namely the close relationship between SC-related bone deficit and circumference.

In conclusion, this study has demonstrated that differential SCIrelated bone deficits at the various anatomical sites of the human tibia are unlikely to be due to anatomical variation in bone mass, but rather are accounted for by variation in periosteal and endocortical circumference. It is possible that both endocortical and periosteal bone resorption occur in response to $\mathrm{SCI}$, and that temporal limitation and a reduced surface:volume ratio lead to comparative preservation of diaphyseal bone. Future studies should try to assess these processes in a longitudinal approach, in order to further expand our understanding of bone biology in disuse.

\section{Acknowledgments}

This study was carried out with internal funding from the Manchester Metropolitan University. We are grateful to Emma Foden for her help with recruitment of some of the volunteers, and, of course to the study participants-without their selfless contribution, this work would not have been possible.

\section{References}

[1] Spungen AM, Wang J, Pierson RN, Bauman WA. Soft tissue body composition differences in monozygotic twins discordant for spinal cord injury. J Appl Physiol 2000;88:1310.

[2] Wilmet E, Ismail AA, Heilporn A, Welraeds D, Bergmann P. Longitudinal study of the bone mineral content and of soft tissue composition after spinal cord section. Paraplegia 1995;33:674.

[3] Bauman WA, Spungen AM, Wang J, Pierson Jr RN, Schwartz E. Continuous loss of bone during chronic immobilization: a monozygotic twin study. Osteoporos Int 1999;10:123-7.

[4] Vestergaard P, Krogh K, Rejnmark L, Mosekilde L. Fracture rates and risk factors for fractures in patients with spinal cord injury. Spinal Cord 1998;36:790.

[5] Ragnarsson KT, Sell GH. Lower extremity fractures after spinal cord injury: a retrospective study. Arch Phys MEd Rehabil 1981;62:418-23.

[6] Eser P, Frotzler A, Zehnder Y, Denoth J. Fracture threshold in the femur and tibia of people with spinal cord injury as determined by peripheral quantitative computed tomography. Arch Phys Med Rehabil 2005;86:498-504.

[7] Eser P, Frotzler A, Zehnder Y, Knecht H, Denoth J, Schiessl H. Relationship between the duration of paralysis and bone structure: a PQCT study of spinal cord injured individuals. Bone 2004;34:869-80.

[8] Frotzler A, Berger M, Knecht H, Eser P. Bone steady-state is established at reduced bone strength after spinal cord injury: a longitudinal study using peripheral quantitative computed tomography (pQCT). Bone 2008;43:549-55.

[9] Rubin CT, Lanyon LE. Regulation of bone mass by mechanical strain magnitude. Calcif Tissue Int 1985;37:411.

[10] Rubin CT, Lanyon LE. Kappa Delta Award paper. Osteoregulatory nature of mechanical stimuli: function as a determinant for adaptive remodeling in bone. J Orthop Res 1987;5:300-10.

[11] Frost HM. Bone "mass" and the "mechanostat": a proposal. Anat Rec 1987;219: $1-9$.

[12] Özkaya N, Nordin M. Fundamentals of biomechanics. New York: Springer; 1998.

[13] Rittweger J. Physiological targets of artificial gravity: adaptive processes in bone. In: Clement G, Bukley A, editors. Artificial Gravity. Berlin: Springer; 2007. p. 191-231.

[14] Schiessl H, Frost HM, Jee WS. Estrogen and bone-muscle strength and mass relationships. Bone 1998;22:1-6.

[15] Rittweger J, Beller G, Ehrig J, Jung C, Koch U, Ramolla J, et al. Bone-muscle strength indices for the human lower leg. Bone 2000;27:319-26.

[16] Rittweger J, Gerrits K, Altenburg T, Reeves N, Maganaris CN, de Haan A. Bone adaptation to altered loading after spinal cord injury: a study of bone and muscle strength. J Musculoskel Neuron Interact 2006;6:269-76.

[17] Rittweger J, Simunic B, Bilancio G, Gaspare DeSanto N, Cirillo M, Biolo G, et al. Bone loss in the lower leg during 35 days of bed rest is predominantly from the cortical compartment. Bone 2009;44:612-8.

[18] Han ZH, Palnitkar S, Rao DS, Nelson D, Parfitt AM. Effects of ethnicity and age or menopause on the remodeling and turnover of iliac bone: implications for mechanisms of bone loss. J Bone Miner Res 1997;12:498-508.

[19] Parfitt AM. Misconceptions (2): turnover is always higher in cancellous than in cortical bone. Bone 2002;30:807-9.

[20] Capozza R, Feldman S, Mortarino P, Reina P, Schiessl H, Rittweger J, et al. Structural analysis of the human tibia by tomographic scans. J Anat 2010;20:1-12.

[21] Rittweger J, Michaelis I, Giehl M, Wüseke P, Felsenberg D. Adjusting for the partial volume effect in cortical bone analyses of pQCT images. Journal of Musculoskeletal Neuron Interactions 2004;4:436.

[22] Ward KA, Adams JE, Hangartner TN. Recommendations for thresholds for cortical bone geometry and density measurement by peripheral quantitative computed tomography. Calcif Tissue Int 2005;77:275-80.

[23] Rittweger J, Beller G, Armbrecht G, Mulder E, Buehring B, Gast U, et al. Prevention of bone loss during 56 days of strict bed rest by side-alternating resistive vibration exercise. Bone 2010:137-47 PMID: 19732856.

[24] Crawley MJ. The R book. Chichester, Sussex, UK: Wiley; 2007.

[25] Garland DE, Adkins RH, Scott M, Singh H, Massih M, Stewart C. Bone loss at the os calcis compared with bone loss at the knee in individuals with spinal cord injury. J Spinal Cord Med 2004;27:207-11.

[26] Riggs BL, Melton III ILJ, Robb RA, Camp JJ, Atkinson EJ, Peterson JM, et al. Population-based study of age and sex differences in bone volumetric density, size, geometry, and structure at different skeletal sites. J Bone Miner Res 2004;19: 1945.

[27] Wilks DC, Winwood K, Gilliver SF, Kwiet A, Sun LW, Gutwasser C, et al. Agedependency in bone mass and geometry: a pQCT study on male and female master sprinters, middle and long distance runners, race-walkers and sedentary people. J Musculoskelet Neuronal Interact 2009;9:236-46.

[28] Schriefer JL, Warden SJ, Saxon LK, Robling AG, Turner CH. Cellular accommodation and the response of bone to mechanical loading. J Biomech 2005;38:1838-45.

[29] Noble BS, Peet N, Stevens HY, Brabbs A, Mosley JR, Reilly GC, et al. Mechanical loading: biphasic osteocyte survival and targeting of osteoclasts for bone destruction in rat cortical bone. Am J Physiol Cell Physiol 2003;284:C934-43.

[30] Frotzler A, Coupaud S, Perret C, Kakebeeke TH, Hunt KJ, Donaldson Nde NC, Eser P. High-volume FES-cycling partially reverses bone loss in people with chronic spinal cord injury. Bone 2008;43:169-76. 
[31] Mohr T, Podenphant J, Biering-Sorensen F, Galbo $\mathrm{H}$, Thamsborg G, Kjaer $\mathrm{M}$. Increased bone mineral density after prolonged electrically induced cycle training of paralyzed limbs in spinal cord injured man. Calcif Tissue Int 1997;61:22.

[32] Tervo T, Nordstrom P, Neovius M, Nordstrom A. Reduced physical activity corresponds with greater bone loss at the trabecular than the cortical bone sites in men. Bone 2009;45:1073-8.
[33] Lang T, LeBlanc A, Evans H, Lu Y, Genant H, Yu A. Cortical and trabecular bone mineral loss from the spine and hip in long-duration spaceflight. J Bone Miner Res 2004;19:1006-12.

[34] Rittweger J, Winwood K, Seynnes O, de Boer M, Wilks D, Lea R, et al. Bone loss from the human distal tibia epiphysis during 24 days of unilateral limb suspension. J Physiol 2006;577:331-7. 